\section{La participación del personal auxiliar de odontología en los sistemas locales de salud ${ }^{1}$}

\author{
Paulo Frazão ${ }^{2}$ \\ y Roberto A. Castellanos ${ }^{2}$
}

\section{RESUMEN}

Aunque numerosos sistemas locales de salud (SILOS) del Brasil emplean personal auxiliar de odontología, existe poca información disponible sobre la contribución de esos trabajadores a los programas de salud bucodental. El objetivo de esta investigación fue analizar la participación de ese personal en 10 SILOS de cinco municipios del estado de São Paulo. Se preparó un cuestionario que fue contestado por 245 (75,4\%) de los 325 auxiliares y técnicos de higiene dental empleados en esos sistemas. Los resultados indicaron que la participación del personal auxiliar de odontología en las actividades de promoción de la salud bucodental es variable en los distintos SILOS estudiados. En algunos, el personal dedicó más tiempo a las actividades de promoción que a las tareas de asistencia odontológica. Las actividades más frecuentes de promoción de la salud bucodental fueron los enjuagues de flúor, la detección de la placa bacteriana dental seguida del cepillado supervisado y las actividades educativas en unidades básicas de salud y escuelas. En todos los casos, la participación del personal auxiliar de odontología de los SILOS ha sido muy importante pues ha contribuido a transformar la práctica de la odontología en el ámbito de la salud pública.

Parte de la tesis de maestría presentada a la Facultad de Salud Pública de la Universidad de São Paulo, Brasil, el 1 de noviembre de 1995.

2 Universidad de São Paulo, Facultad de Salud Pública, São Paulo, Brasil. Dirección postal: Av Dr Arnaldo, 715, CEP 01246-904, São Paulo, Brasil. Dirección electrónica: pafrazao@usp.br
La meta más importante de las políticas de salud pública en el mundo durante el presente siglo es alcanzar la salud para todos en el año 2000 (1). La estrategia utilizada es la atención primaria (2) en el ámbito de los sistemas locales de salud (SILOS) (3), la cual está basada en el concepto de promoción de la salud expresado en la Carta de Ottawa. Esta Carta, que fue adoptada por los delegados de 38 países a la primera Conferencia Internacional sobre la Promoción de la Salud (Ottawa, 1986), declaró como propósito de dicha actividad fortalecer y capacitar a las personas y colectividades (como municipios, asociaciones, escuelas, entidades comerciales e industriales, organizaciones de trabajadores y medios de comunicación) para que sepan controlar los factores que determinan la salud y la enfermedad y mejorar los niveles de salud. Desde el punto de vista estratégico, la meta consiste en identificar los factores que obstaculizan las políticas públicas de salud y superarlos mediante la reorientación de los servicios de atención, diversas acciones intersectoriales y actividades colectivas y comunitarias.

Desde los años ochenta, el gobierno del Brasil se esfuerza por descentralizar las actividades de salud y organizar los SILOS. Se trata de un proceso social (4) con aspectos políticos, normativos, gerenciales y operacionales, entre otros, que produjo tres estructuras diferentes: 1) SILOS que abarcan la misma base territorial, poblacional, administrativa y sanitaria que el municipio; 2) SILOS que abarcan una parte del municipio (generalmente de densidad poblacional mediana y alta), y 3) SILOS que incluyen varios municipios pequeños que se comunican fácilmente entre sí. La situación creó condiciones que llevaron a una modificación de las actividades de odontología dirigidas a promover la salud colectiva. Por ejemplo, en algunas localidades se preparó al personal auxiliar de odontología (PAO) para participar en actividades de promoción de la salud bucodental y de asistencia odontológica individual, de acuerdo con las formulaciones sanitarias previstas para la región (5).

La reglamentación nacional de la profesión definió cuatro tipos de personal auxiliar: el técnico en higiene dental (THD) y el técnico de prótesis dental, ambos con estudios secundarios; el auxiliar de consultorio dental (ACD) y el auxiliar de prótesis dental, ambos de nivel primario o con 8 años de estudio (6). En la presente investigación, la expre- 
sión personal auxiliar odontológico (PAO) se refiere solamente a los ACD y THD y, excluye, por lo tanto, al personal de laboratorios de prótesis.

Si bien existe poca información sobre el tema, se admite que la participación de ese personal en los servicios odontológicos del Brasil sigue siendo pequeña, pero que ha aumentado en los últimos 10 años. Según los datos nacionales de 1993, la razón PAO: cirujanos dentistas (CD) es cercana a 1:10, con probable subregistro del personal auxiliar (6). La razón THD:CD era cercana a 2:100, cantidad bien modesta si se la compara con la razón higienista dental (HD):CD en países como Corea (8:10), Canadá (6:10), Estados Unidos de América (55:100), el Japón (5:10), Suecia (2:10) y el Reino Unido de Gran Bretaña e Irlanda del Norte (12:100) (7).

De modo general, se estima que la mayoría del PAO empleado en el Brasil es joven, pertenece al sexo femenino y tiene un nivel de escolaridad insatisfactorio para ejercer las funciones que se le asignan, pues menos de $30 \%$ de las mujeres brasileñas cursan 8 o más años de estudios (8). Además, los salarios son bajos cuando se los compara con los de los HD de los servicios de salud pública de Estados Unidos (9).

El propósito de esta investigación fue describir algunas características del PAO empleado en 10 SILOS de cinco municipios del Estado de São Paulo en 1994 y analizar su participación en las actividades de promoción de la salud bucodental y de asistencia individual. Las hipótesis enunciadas fueron las siguientes: 1) la fracción estimada de la jornada de trabajo que el PAO dedica a actividades de promoción de la salud bucodental es menor que la fracción dedicada a las actividades de atención individual; 2) la fracción estimada de la jornada de trabajo que el PAO dedica a actividades de promoción de salud bucodental se ve influenciada por el municipio y los SILOS que lo emplean.

\section{MATERIALES Y MÉTODOS}

Sobre la base de los datos proporcionados por los coordinadores de programas de salud bucodental se identificaron cinco municipios (A, B, C, D, E) cuyo PAO estuvo empleado durante más de 5 años (muestra intencionada). A continuación se solicitó la autorización de los directores de los SILOS de esos municipios para reunir al PAO durante la jornada de trabajo con el fin de responder a un cuestionario. Se eligió ese procedimiento a fin de facilitar la recolección de los datos, garantizar el mayor número de respuestas y evitar que el intercambio de información entre el personal participante afectara a las respuestas. Solamente un SILOS del municipio E negó la autorización.
Para elaborar el cuestionario se realizó un estudio exploratorio (10) destinado a adecuar el lenguaje de este instrumento de observación y controlar la subjetividad del investigador. Las variables estudiadas fueron municipio y SILOS donde se trabajaba, sexo, edad, función, duración de la jornada de trabajo, salario, escolaridad, formación profesional, tipo de local de trabajo y participación en actividades de promoción de la salud bucodental y de atención odontológica individual. Las actividades de promoción de la salud bucodental investigadas fueron los enjuagues de flúor; la detección de la placa bacteriana dental y cepillado de dientes supervisado; aplicación tópica de flúor con cepillo o cubeta, y actividades educativas. Se registraron la frecuencia de estas actividades y el número de personas beneficiadas. Las actividades de atención individual investigadas fueron la aplicación tópica de flúor, sellantes y diaminofluoreto de plata $\left[\left(\mathrm{NH}_{3}\right)_{2} \mathrm{AgF}\right]$; el detartraje (eliminación de la placa bacteriana) y pulido coronario y la inserción de materiales restauradores provisorios o definitivos.

Una vez aprobado, se aplicó el cuestionario en los meses de junio, agosto y septiembre de 1994. La distribución del tiempo de la jornada de trabajo del PAO dedicado a las actividades de promoción de la salud bucodental y a la atención individual se estimó mediante los cinco parámetros presentados en el anexo, sobre la base de la frecuencia de las actividades y el número de personas beneficiadas (11).

\section{RESULTADOS}

Las diferencias entre los municipios fueron notables desde el punto de vista de la economía y de la razón horas de trabajo PAO:CD. El cuadro 1 presenta algunas características de los cinco municipios donde se localizaban los 10 SILOS estudiados. Los resultados se refieren a 245 trabajadores $(75,4 \%)$ que devolvieron el cuestionario completamente lleno. De estos, 153 declararon ejercer funciones de auxiliar de consultorio dental (ACD) y 92 , de técnico en higiene dental (THD) (cuadro 2).

La mayor parte del PAO empleado en los 10 SILOS eran mujeres $(92,7 \%)$ jóvenes cuyas edades oscilaban entre los 17 y los 37 años. Aproximadamente $95 \%$ del total había cursado la escuela primaria completa y $70 \%$ había terminado los estudios secundarios. La figura 1 indica que la mayoría del PAO de cuatro de los cinco municipios estudiados había completado su formación técnica específica o estaba en vías de completarla. Los datos desagregados por tipo de personal indican que la proporción de trabajadores con calificación profesional era más alta entre los $\mathrm{ACD}(61,4 \%)$ que entre los THD $(25 \%)$. El municipio D, que reunía cuatro de los 10 SILOS 
CUADRO 1. Características de los municipios del estudio. São Paulo, Brasil, 1994

\begin{tabular}{|c|c|c|c|c|c|}
\hline \multirow[b]{2}{*}{ Características } & \multicolumn{5}{|c|}{ Municipios } \\
\hline & A & B & C & $\mathrm{D}$ & E \\
\hline Relación SILOS estudiados/existentes & $1 / 1$ & $1 / 1$ & $1 / 1$ & $4 / 4$ & $3 / 4$ \\
\hline Habitantes $^{\mathrm{a}}$ & 100000 & 200000 & 48000 & 442000 & 846000 \\
\hline Tipo de economía & Agropecuaria & $\begin{array}{l}\text { Ciudad- } \\
\text { dormitorio }\end{array}$ & Agropecuaria & Industria & $\begin{array}{c}\text { Industria y } \\
\text { servicios }\end{array}$ \\
\hline Distancia hasta la capital (Km) & 120 & 30 & 500 & 84 & 100 \\
\hline Población urbana con agua tratada (\%) & 100 & 85 & $\ldots \mathrm{b}$ & 90 & 98 \\
\hline Población urbana con alcantarillado (\%) & 96 & 15 & $\ldots$ & 75 & 86 \\
\hline Horas de trabajo (PAO:CD) & $40: 10$ & $20: 10$ & $50: 10$ & $17: 10$ & $8: 10$ \\
\hline
\end{tabular}

Fuente: Directorio de salud de cada municipio.

a Número aproximado de habitantes.

${ }^{b}$ Los puntos significan que no hay datos disponibles.

CUADRO 2. Distribución del personal auxiliar odontológico empleado que respondió al cuestionario, según la función que ejerce en los SILOS del estudio. São Paulo, Brasil, 1994

\begin{tabular}{|c|c|c|c|c|c|c|}
\hline \multirow[b]{3}{*}{ SILOS } & \multicolumn{6}{|c|}{ Función } \\
\hline & \multicolumn{3}{|c|}{$A C D^{a}$} & \multicolumn{3}{|c|}{$\mathrm{THD}^{\mathrm{b}}$} \\
\hline & $E^{c}$ & $N^{d}$ & $\%$ & E & $\mathrm{N}$ & $\%$ \\
\hline A & 40 & 31 & 77,5 & 7 & 7 & 100,0 \\
\hline B & 21 & 13 & 61,9 & 13 & 10 & 76,9 \\
\hline$C$ & 12 & 11 & 91,7 & 7 & 6 & 85,7 \\
\hline D1 & 21 & 16 & 76,2 & 21 & 14 & 66,7 \\
\hline D2 & 11 & 10 & 90,9 & 12 & 8 & 66,7 \\
\hline D3 & 17 & 14 & 82,4 & 33 & 26 & 78,8 \\
\hline D4 & 9 & 6 & 66,7 & 22 & 21 & 95,5 \\
\hline E1 & 46 & 25 & 54,3 & $\ldots$ & $\ldots$ & $\ldots$ \\
\hline E2 & 17 & 15 & 88,2 & $\ldots$ & $\ldots$ & $\ldots$ \\
\hline E3 & 16 & 12 & 75,0 & $\ldots$ & $\ldots$ & $\ldots$ \\
\hline Total & 210 & 153 & 72,9 & 115 & 92 & 80,0 \\
\hline
\end{tabular}

a Auxiliar de consultorio dental: personal auxiliar de nivel básico.

b Técnico en higiene dental: personal técnico de nivel secundario.

c Personal empleado.

d Personal que respondió al cuestionario.

e Los puntos significan que no hay datos disponibles.

en estudio, presentaba la menor proporción de trabajadores calificados.

La población en estudio trabajaba en promedio 40 horas semanales, los ACD recibían US\$215,00 por mes y los THD $\$ 220,00$. En la figura 2 , se puede observar que la variación del salario recibido era mayor entre los ACD que entre los THD. Los sistemas municipales de salud D y E presentaban mayores promedios salariales $\mathrm{y}$, aunque el $\mathrm{mu}-$ nicipio E solo empleaba $\mathrm{ACD}$, los remuneraba con un sueldo casi tres veces mayor que el de los ACD del municipio A y casi el doble del recibido por los THD del mismo municipio.

En la mayoría de los SILOS, la participación del PAO en las actividades de promoción de la salud bucodental era mayor cuando estas se realiza- ban en las escuelas $u$ otros entornos sociales (figura 3) que cuando ocurrían en las unidades básicas de salud (figura 4). Sin embargo, el PAO de los SILOS de los municipios A, B, C y de los SILOS 1, 2 y 3 del municipio $E$ desempeñaba la mayoría de esas actividades en las unidades básicas de salud (figura 4). En general, se observó un gran número de actividades educativas y de detección de placa bacteriana seguida de cepillado supervisado $y$, en menor proporción, de enjuagues de flúor. En los SILOS del municipio E, los enjuagues de flúor se aplicaban a personal vinculado a otros sectores de la administración pública.

Con respecto a la participación en la atención odontológica individual, más de la mitad de los THD (53,3\%) ejercían también las tareas propias de 
FIGURA 1. Porcentaje del personal auxiliar de odontología (auxiliar de consultorio dental ACD y técnico en higiene dental [THD]) con y sin curso de formación profesional específica en el municipio. São Paulo, Brasil, 1994

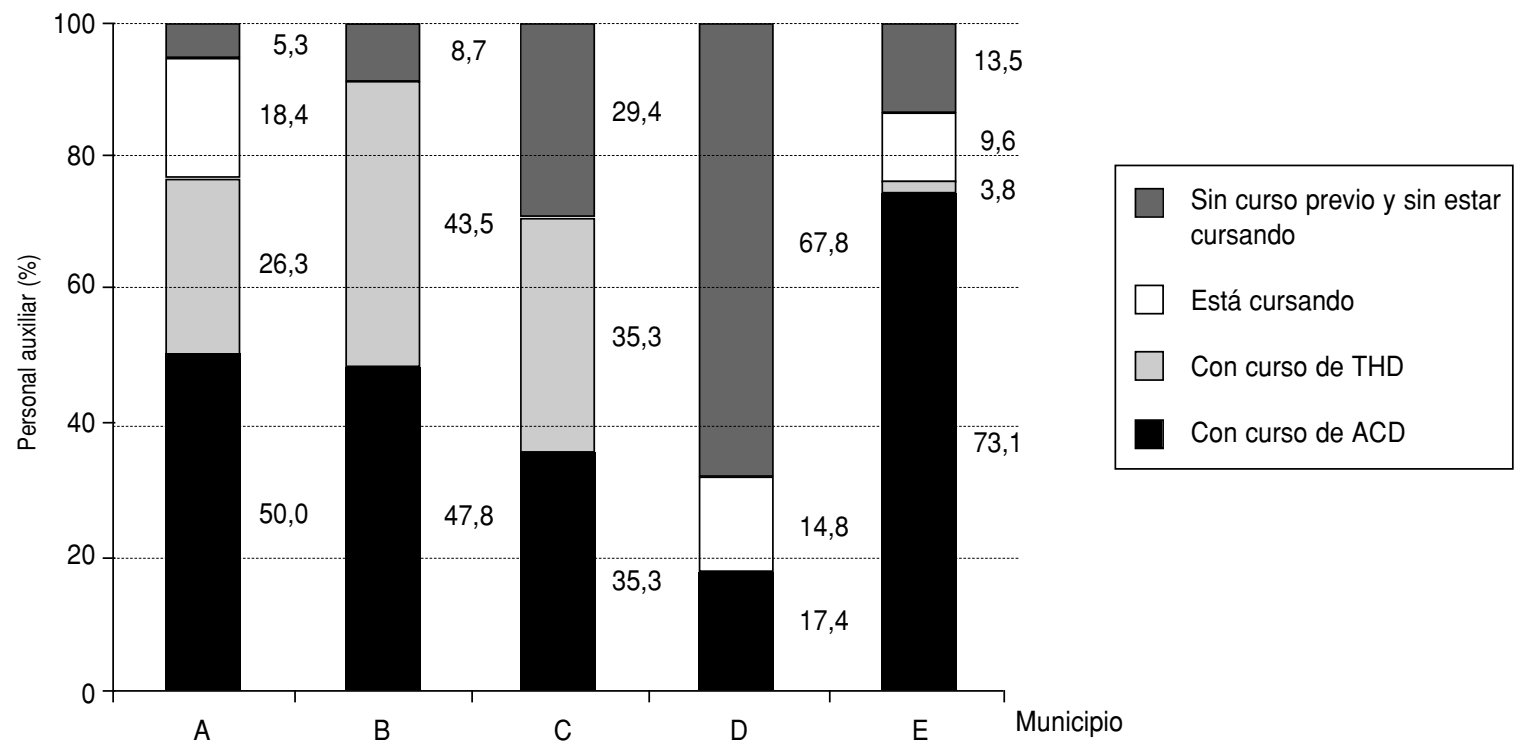

FIGURA 2. Salario promedio declarado por el personal auxiliar de consultorio dental (ACD) y técnico en higiene dental (THD), según función y municipio. São Paulo, Brasil, 1994 (cifras en US\$)

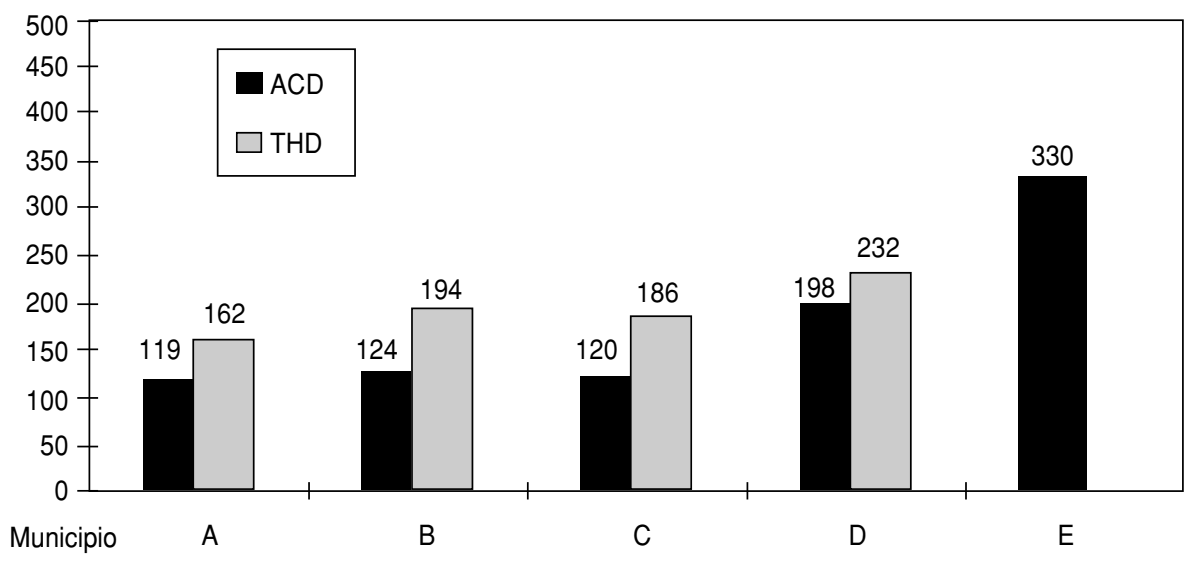

un ACD, tales como preparación de la sala, limpieza, desinfección y esterilización de los instrumentos y preparación de cementos y otros materiales durante la atención clínica de los pacientes. Otro aspecto importante es la pequeña proporción del PAO que practica rutinariamente las técnicas de trabajo a cuatro manos para asistir a los odontólogos en las tareas de atención clínica en los SILOS de los municipios B y C y en los SILOS 1, 2 y 4 del municipio D.
Con respecto a la delegación de funciones en la atención individual, cerca de $20 \%$ de los ACD declararon que realizaban aplicaciones tópicas de flúor. Casi 100\% de los THD de los SILOS A, B, y $\mathrm{C}$ hacían aplicaciones tópicas de flúor y sellantes, realizaban detartraje y pulido coronario, y practicaban la inserción y condensación de materiales restauradores. En el SILOS del municipio A, 14,3\% de los THD aplicaban diaminofluoreto de plata y en los SILOS de los municipios B y C todos los THD 
FIGURA 3. Porcentaje del personal auxiliar de odontología que realiza actividades de promoción de la salud bucodental en escuelas y otros espacios sociales, ${ }^{a}$ según el tipo de actividades por SILOS. São Paulo, Brasil, 1994

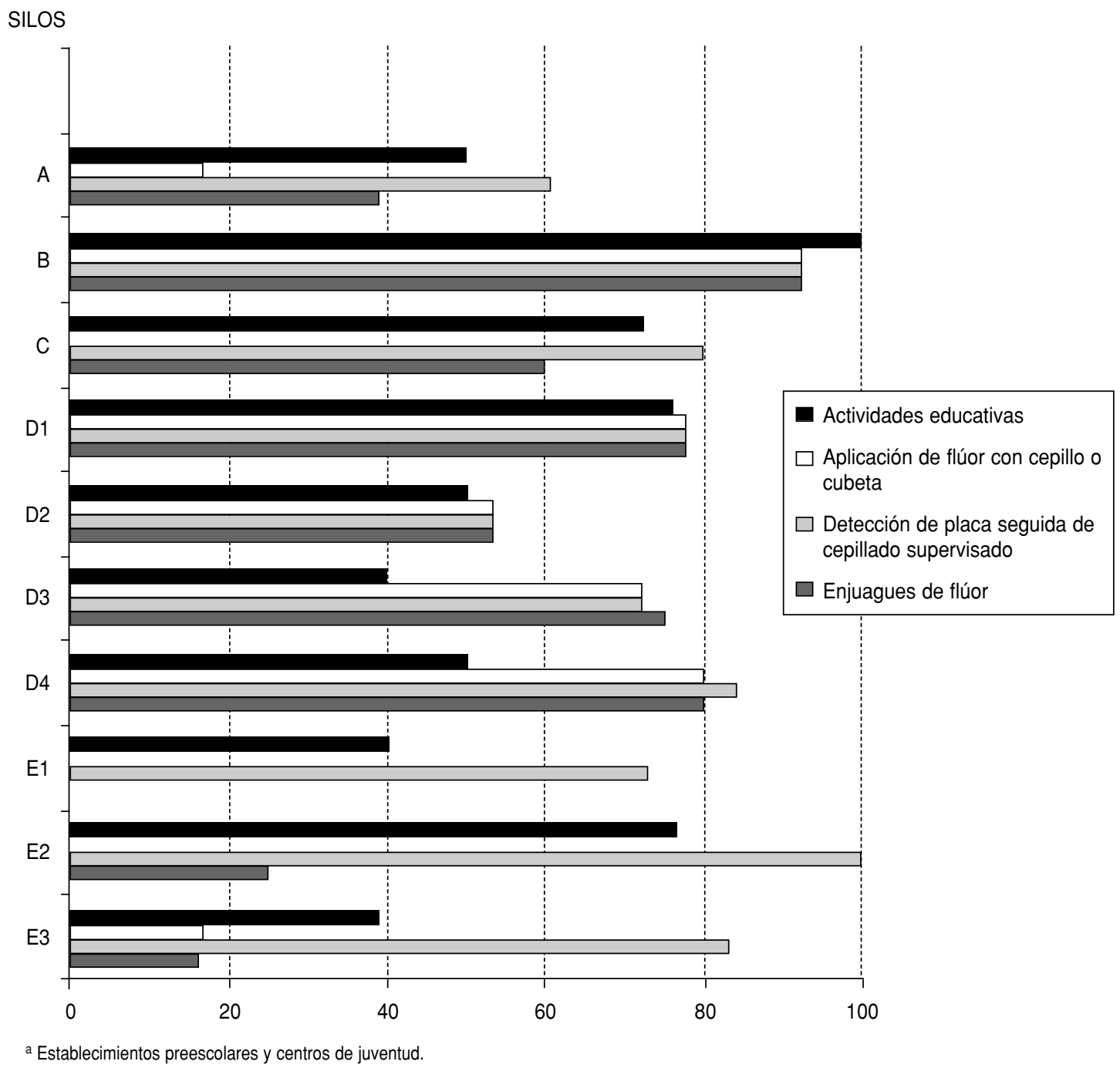

utilizaban ese producto. Solamente $13 \%$ de los THD de los SILOS D1 y D2 aplicaban flúor tópico durante la atención individual. La figura 5 sintetiza la distribución estimada del tiempo de la jornada de trabajo del PAO. En algunas localidades - por ejemplo, B, D2 y D4- el personal dedicaba más tiempo de su jornada de trabajo a la promoción de la salud bucodental que a las actividades de atención individual.

Lo expuesto permite afirmar que las observaciones en los SILOS A, C, D1, D3, E1, E2 y E3 no permiten rechazar la hipótesis 1 y que las observaciones en los SILOS B, D2 y D4 no la respaldan. Los resultados obtenidos no permiten rechazar la hipótesis 2 porque muestran que la participación del
PAO en actividades de promoción de la salud bucodental depende del SILOS y del municipio que emplean a ese personal.

\section{DISCUSIÓN}

Los resultados deben ser interpretados con cautela debido a las características metodológicas del presente estudio: una muestra intencionada de personal auxiliar de odontología que trabaja en 10 SILOS aportó información por medio de respuestas a un cuestionario. El término personal auxiliar de odontología comprende una gran variedad de sujetos con diferente formación, responsabilidades y 
FIGURA 4. Porcentaje de personal auxiliar de consultorio dental (ACD) y técnico en higiene dental (THD) que realiza actividades de promoción de la salud bucodental en unidades básicas de salud, según el tipo de actividades por SILOS. São Paulo, Brasil, 1994

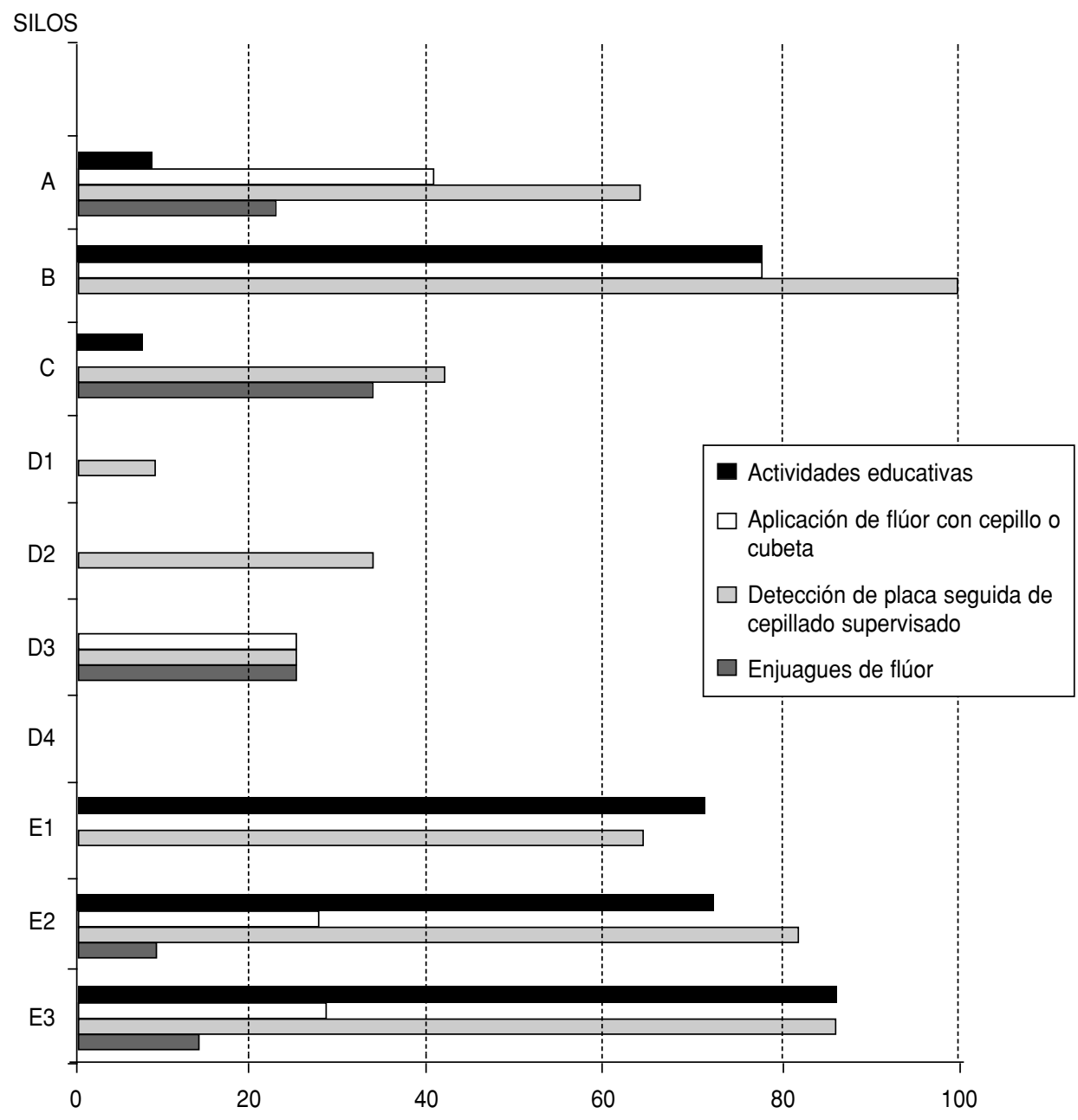

atribuciones, así como diferente reglamentación y condiciones para obtener la licencia laboral conforme a los países, estados, departamentos y unidades político-administrativas menores.

La gran variación del salario recibido por el PAO estudiado permite suponer que su valor estaba más relacionado con las características económicas del municipio (véase el cuadro 1) que con la calificación profesional del personal o el tipo de función que ejercía. A diferencia de lo indicado por los datos nacionales, el PAO empleado en los SILOS investigados gozaba de una escolaridad alta que obedece, muy probablemente, a las políticas de recursos humanos locales aplicadas por los dirigentes durante la selección de ese personal en la institución de salud y después de su ingreso.

Con respecto a la formación profesional específica, se verificó que había más facilidades para ob- tener el certificado de $\mathrm{ACD}$, cuyo curso era más corto, tenía menores requisitos para el ingreso, un costo más bajo y mejor aceptación en las instituciones de salud que el certificado de THD, cuyo currículo era más extenso y de contenido teóricopráctico más complejo.

Es posible que las diferencias encontradas en los SILOS hayan respondido a las distintas estrategias que se aplicaron en el Brasil durante la década de 1980 con el fin de introducir cambios en la organización de la atención de la salud bucodental en los sistemas de salud y en el entrenamiento del PAO. Aunque al iniciarse el período no se daba una preparación formal al personal, al final de la década la implantación de mecanismos de articulación entre los sistemas de salud y los de educación tornó más viable y factible adoptar la estrategia de formación en el servicio. 
FIGURA 5. Porcentaje de tiempo de trabajo que el personal auxiliar de consultorio dental (ACD) y técnico en higiene dental (THD), dedica a las actividades de promoción de la salud bucodental, según SILOS y local de las acciones. São Paulo, Brasil, 1994

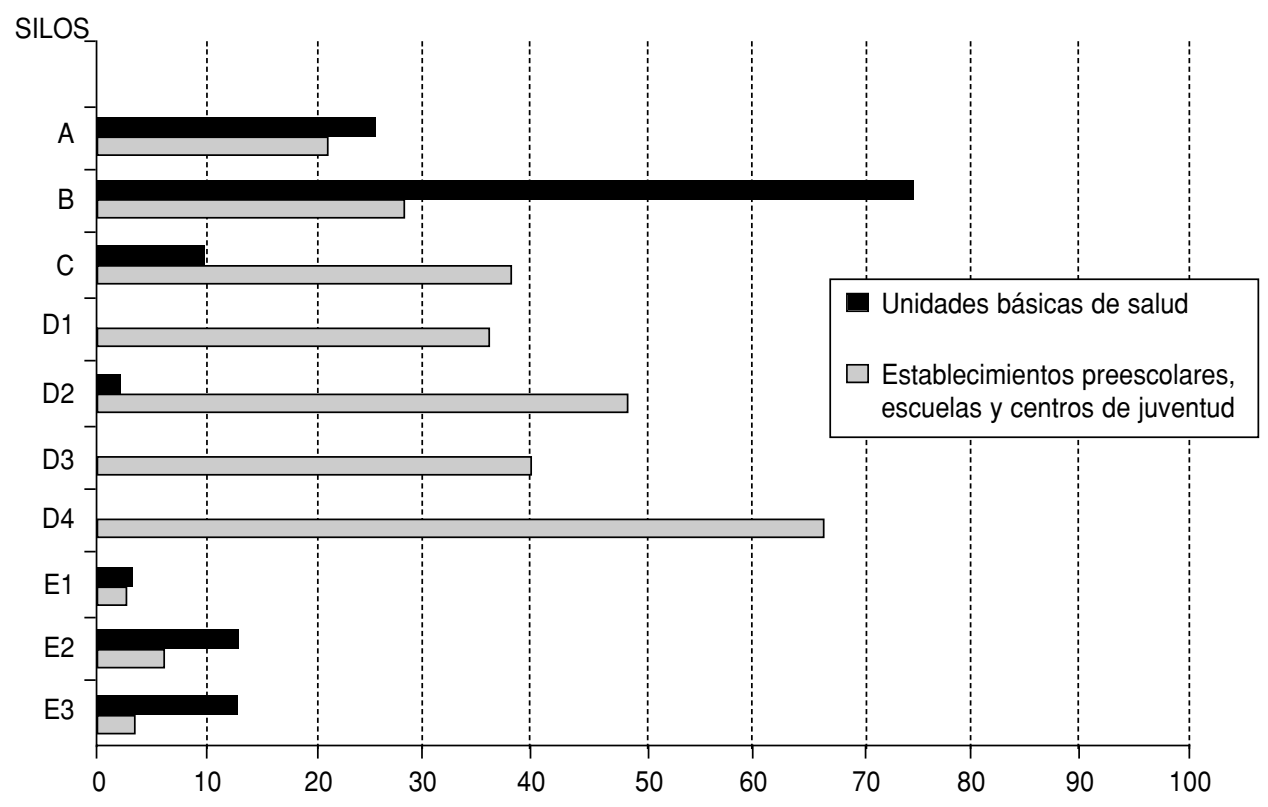

La pequeña proporción del PAO que rutinariamente trabajaba a cuatro manos y la mitad o más de los THD que realizaban tareas propias de los ACD indican que existían insuficiencias cualitativas y cuantitativas de PAO en los SILOS investigados y que era necesario organizar actividades para desarrollar los recursos humanos $(12,13)$. Los resultados indicaron que los SILOS utilizaban de forma distinta a su personal. Los SILOS de los municipios $\mathrm{A}, \mathrm{B}$ y $\mathrm{C}$ delegaban en los THD las funciones relacionadas con la atención odontológica individual con mayor frecuencia que los SILOS de los municipios D y E. Además, pese a que la mayoría de los SILOS del país no emplean THD y que la razón PAO:CD es cercana a 1:10 — característica de un modelo de baja cobertura y alto costo- - se observó una notable participación del $\mathrm{PAO}$ en actividades de promoción de la salud bucodental en tres de los 10 SILOS investigados. Esos resultados son semejantes a los encontrados por Deal (9). De modo similar, hay territorios y países que difieren en su utilización del PAO. Según Rock y Bradnock (14), los odontólogos del Reino Unido delegaban a los HD más actividades de raspado y pulido coronario. En Polonia, Janczuk (15) verificó que cerca de $45 \%$ de los HD realizaban actividades de educación en salud bucodental y de profilaxis con flúor y que solamente $25 \%$ desarrollaban actividades de detartraje e inserción de amalgama. Axelsson (16) informó que los HD en Suecia se utilizan principalmente para fomentar un mayor cuidado a la propia salud entre las personas y los grupos; realizar procedimientos de raspado radicular, limpieza mecánica profesional, aplicación de pruebas salivales, geles, varnices y selladores con flúor; finalizar las restauraciones; remover los excesos y prominencias y dar orientaciones dietéticas. Así, el trabajo puede enfocar más a las actividades clínicas (14-23) o las de promoción de la salud bucodental $(9,24,25)$,

En la última década, varios analistas han reiterado en seminarios y publicaciones del Brasil (27-30) y América Latina (31) que para mejorar los niveles de salud bucodental se deben trazar dos objetivos estratégicos: aumentar las medidas de prevención y promoción de la salud bucodental e incorporar y preparar al PAO de los servicios de salud. Desde el punto de vista de la salud pública, eso sugiere que debe observarse el principio general de "prestar el mayor beneficio para el mayor número posible de personas", que significa "procurar un beneficio razonable para el mayor número en vez de un beneficio total para un pequeño número" (26), a modo de lograr un equilibrio entre la extensión (población beneficiada) y la profundidad (tipos de servicios prestados) de los programas de salud.

Como consecuencia, el empleo del PAO debe orientarse de forma coordinada tanto a las actividades de promoción de la salud bucodental — cuya finalidad es disminuir la prevalencia y gravedad de las enfermedades bucodentales en la población- 
como a las actividades de tratamiento. Si bien estos aspectos plantean problemas que no son objeto del presente artículo, constituyen desafíos fundamentales para los dirigentes, trabajadores y usuarios del proceso de construcción de los SILOS.

En los países industrializados, la caries dental y las enfermedades periodontales están disminuyendo y los programas de salud bucodental se dirigen a los niños y a la población adulta y anciana (32); en cambio, la prevalencia de las principales enfermedades bucodentales y las medidas para enfrentarlas varían bastante en los países en desarrollo. En el Brasil se observa una tendencia a la disminución de la caries dental en la infancia (33) que puede atribuirse a la fluoración de muchos sistemas de abastecimiento de agua y, más recientemente, a la adición de flúor a las cremas dentales. Con relación al personal de nivel universitario, el país presenta una razón CD:población de 1:1142, con una tendencia al aumento anual desproporcionado del número de odontólogos en relación con el de la población (incremento de CD: 5,92\%; incremento de habitantes: $1,82 \%$ ) y al mantenimiento del desequilibrio de su distribución en las diferentes partes del país. Por lo tanto, es de gran importancia reorientar los recursos humanos de odontología. Los resultados del presente estudio muestran modificaciones en las características de la odontología en el ámbito de la salud pública en algunos SILOS donde había una mayor utilización del PAO en las actividades de promoción de la salud bucodental.

Agradecimiento. Se agradece a los trabajadores que ejercen funciones auxiliares en los servicios odontológicos, razón y fuente primaria de esta investigación; en particular a los auxiliares y técnicos en higiene dental de los SILOS que respondieron el cuestionario con prontitud y participaron en la investigación.

\section{REFERENCIAS}

1. Organización Panamericana de la Salud. Salud para todos en el año 2000. Estrategias. Washington, D.C.: OPS; 1980

2. Organización Mundial de la Salud. Atención primaria de salud: informe de la Conferencia Internacional sobre Atención Primaria de Salud. Alma-Ata, URSS, 6-12 de septiembre de 1978. Ginebra: OMS; 1978. (Serie Salud para Todos 1).

3. Organización Panamericana de la Salud. Los sistemas locales de salud: aspectos conceptuales y metodológicos. Washington, DC: OPS; 1988.

4. Mendes EV, org. Distrito sanitário: o processo social de mudança das práticas sanitárias do Sistema Único de Saúde. São Paulo-Rio de Janeiro: Hucitec/Abrasco; 1994.

5. Gillespie GM, Yépez P. La salud oral: un componente de los sistemas locales de salud. En: Paganini JM, Capote Mir R. Los sistemas locales de salud: conceptos, métodos, experiencias. Washington, D.C.: Organización Panamericana de la Salud; 1990. (Publicación Científica 519).

6. Brasil, Conselho Federal de Odontologia. Força de trabalho. Rio de Janeiro: CFO; 1993.

7. World Dental Federation. Basic Facts 1990: Dentistry around the world. London: IDF; 1990.

8. Brasil, Secretaría de Planejamento da Presidencia da República; Brasil, Instituto Brasileiro de Geografia e Esta- tistica. Anuário estatístico do Brasil 1996. Rio de Janeiro: FIBGE; 1996.

9. Deal TS. The role of dental hygienists on state public health programs. J Dent Hyg 1990;64(6):286-289.

10. Piovesan A, Temporini ER. Pesquisa exploratória: procedimento metodológico para o estudo de fatores humanos no campo da saúde pública. Rev Saude Publica 1995; 29(4):318-325.

11. Frazão P. A participação do pessoal auxiliar odontológico em dez sistemas locais de saúde de cinco municípios no Estado de São Paulo, 1994. [Dissertação de Mestrado]. São Paulo: Faculdade de Saúde PúblicaUSP; 1995

12. Da Silva JA. Desarrollo de recursos humanos para los sistemas locales de salud: Brasil. Educ Med Salud 1993; 27(1):32-49.

13. Frazão P. Desenvolvimento de pessoal universitário odontológico na perspectiva do Sistema Único de Saúde. Rev Saúde em Debate 1994; 42:30-35.

14. Rock WP, Bradnock G. The employment of dental hygienists within the General Dental Service in the United Kingdom. Br Dent J 1976;140(10): 351-352.

15. Janczuk Z. Dental hygienists in Poland. Community Dent Oral Epidemiol 1978;6(6):283-285.

16. Axelsson P, Rolandsson $M$, Bjerner B. How swedish dental hygienists apply their training program in the field. Community Dent Oral Epidemiol 1993;21(5):297-302.

17. Steele LP. Dental nurses in Trinidad and Tobago. Bull Pan Am Health Organ 1988;22(4):364-372.

18. Cirincione UK, Wils WJ. Survey of dental hygienists in the hospital setting. J Dent Hyg 1990;64(5):239-245.

19. Turner PJ, Pinson RR. Training hygienists for an auxiliary role in orthodontics. Br Den J 1993;175(6): 209-213.

20. Qualtrough AJ, Piddock V. Dental education in Japan. Br Dent J 1993; 174(3):111-112.

21. Pack AR. Hygienists and their role in dental practice. N Z Dent J 1995;91 (404):57-61.

22. Wexler G. Dental hygienists in Australia and their employment in orthodontic practice. Br J Orthod 1995; 22(1):98-100.

23. Fernández Ollero A, Cordero MA, Castaño A, Casado A, González A. Actitudes y conocimientos del personal auxiliar ante la prevención en odontología: resultados de una encuesta. Rev Act Odontoestomat Esp 1995;55:44-52.

24. Schou L, Tronbjerg C, Holst I. A national survey of dental hygienists in Denmark. Community Dent Health 1986;3(1):91-98.

25. Laiho M, Honkala E, Kannas L. How is oral health education conducted in Finnish health centers? Community 
Dent Oral Epidemiol 1995;23(2): 119-24.

26. Chaves MM. Manual de odontologia sanitária. São Paulo, Massao Ohno, 1960.

27. Viegas AR. A importância do Técnico em Higiene Dental na assistência odontológica em São Paulo. [Conferência na Sessão Solene de Abertura do Curso de Técnico em Higiene Dental da Secretaria de Higiene e Saúde da Prefeitura do Município de São Paulo]. São Paulo: 1988.

28. Narvai PC, Manfredini MA, Botazzo C, Raineri N, Schneider D, Frazão P. Contra o técnico em higiene dental. Rev Saude Debate 1990; 28: 59-65.

29. Rosa AGF, Souza DS, Schneider Filho DA, Lopes ES, Uchôa HW, Toledo JP, Narvai PC, Fernandes RAC, Gevaerd SP, Chianca, TZ. O que fazer nos municípios? Rio de Janeiro: Rede Cedros; 1992. (Cadernos de Saúde Bucal 1).

30. Brasil, Ministério da Saúde. Segunda Conferência Nacional de Saúde Bucal: relatorio final. Brasília: Ministério da Saúde; 1993.

31. Organización Panamericana de la Salud. Desarrollo y fortalecimiento de los sistemas locales de salud en la transformación de los sistemas na- cionales de salud: la salud bucal. Washington, DC: OPS; 1993. (Serie HSD/SILOS 22).

32. World Health Organization, Oral Health Unit. Oral health for the 21st century. Geneva: WHO; 1994.

33. Pinto VG. Indice de cárie no Brasil e no mundo. RGO 1996;44(1):8-12.

Manuscrito recibido el 15 de agosto de 1997 y aceptado para publicación en versión revisada el 9 de diciembre de 1998.

\section{ANEXO 1. Método para estimar las horas de trabajo mensuales dedicadas a las actividades de promoción de la salud bucodental y a la atención individual}

1. Número de personas beneficiadas por mes:

Se establecieron la frecuencia con que el personal auxiliar de odontología se dedicó a la actividad de promoción y el número de personas beneficiadas en cada tipo de local. Los valores se ajustaron multiplicando la cantidad por cuatro cuando la información correspondía a una semana y dividiendo la cantidad por 12 cuando la información correspondía a un año;

2. Promedio de las personas beneficiadas por el trabajo del personal auxiliar (ACD y THD): Se dividió el total de personas beneficiadas por mes por la cantidad de personal auxiliar que realizaba las actividades de promoción en cada SILOS;

3. Parámetros de rendimiento medio:

Se elaboró la información provista por los coordinadores y supervisores de las actividades del personal auxiliar (véase el cuadro) teniendo en cuenta el tipo de actividad y el local donde se realizaron;

Parámetros de rendimiento medio del personal auxiliar de odontología. São Paulo, Brasil, 1994

\begin{tabular}{lcc}
\hline & \multicolumn{2}{c}{ Locales donde se realizan las actividades } \\
\cline { 2 - 3 } \multicolumn{1}{c}{ Tipo de actividad } & $\begin{array}{c}\text { Establecimientos } \\
\text { preescolares, escuelas } \\
\text { y centros de juventud }\end{array}$ & $\begin{array}{c}\text { Unidades básicas } \\
\text { de salud }\end{array}$ \\
\hline Enjuagues de flúor & 125 & - \\
Detección de placa seguida de cepillado supervisado & 30 & 4 \\
Aplicación de flúor con cepillo o cubeta & 15 & 3 \\
\hline
\end{tabular}

Nota: Las diferencias obedecen a las características de la estructura física de cada local y a la manera en que se realizan las actividades en cada uno. Las cifras representan el número de actividades realizadas en un mes.

4. Número de horas mensuales dedicadas a cada actividad:

Se dividió el promedio de las personas beneficiadas mensualmente por cada auxiliar por el parámetro establecido para esa actividad de acuerdo con el tipo de local donde se realizó la misma; 
5. Total de horas mensuales dedicadas a actividades colectivas:

Se sumaron las horas mensuales dedicadas a cada actividad colectiva o de promoción de la salud bucodental, en las que ya estaban incluidas las actividades educativas.

Con los datos así obtenidos fue posible estimar el tiempo de su jornada de trabajo que el PAO de cada SILOS dedica a las actividades colectivas. Se consideró que el tiempo restante estaba dedicado a actividades de atención odontológica individual.

Con anterioridad a la presente investigación, el país no contaba con estudios de rendimiento medio para los diferentes tipos de actividades colectivas que sirvieran para orientar el cálculo de las estimaciones descritas más arriba. Debe señalarse que las inferencias tienen solamente validez interna debido a que los parámetros obtenidos fueron elaborados para facilitar la comparación entre los diferentes SILOS.

ABSTRACT Though numerous local health systems (sistemas locales de salud, or SILOS) in Brazil employ dental assistants, there is little information on the contributions these workers make to oral health programs. The purpose of this study was to examine the role of such workers in 10 SILOS in five municipalities in the state of São Paulo. Of the 325 dental assistants and dental hygienists employed in those systems, 245 (75.4\%) answered a questionnaire that had been prepared. The results showed variations in the degree to which dental assistants participated in oral health promotion activities in the SILOS studied. In some SILOS, these workers devoted more time to dental health promotion activities than to helping perform dental tasks with individual patients. The most frequent oral health promotion activities were fluoride rinses, plaque detection followed by supervised brushing, and educational activities at basic health units and schools. In all cases, dental assistants working in the SILOS played a significant role in helping transform the practice of dentistry within the sphere of public health. 